\title{
IIIROSOOPY TOPAY
}

คPRIL I 997

ISSUE \#97-5

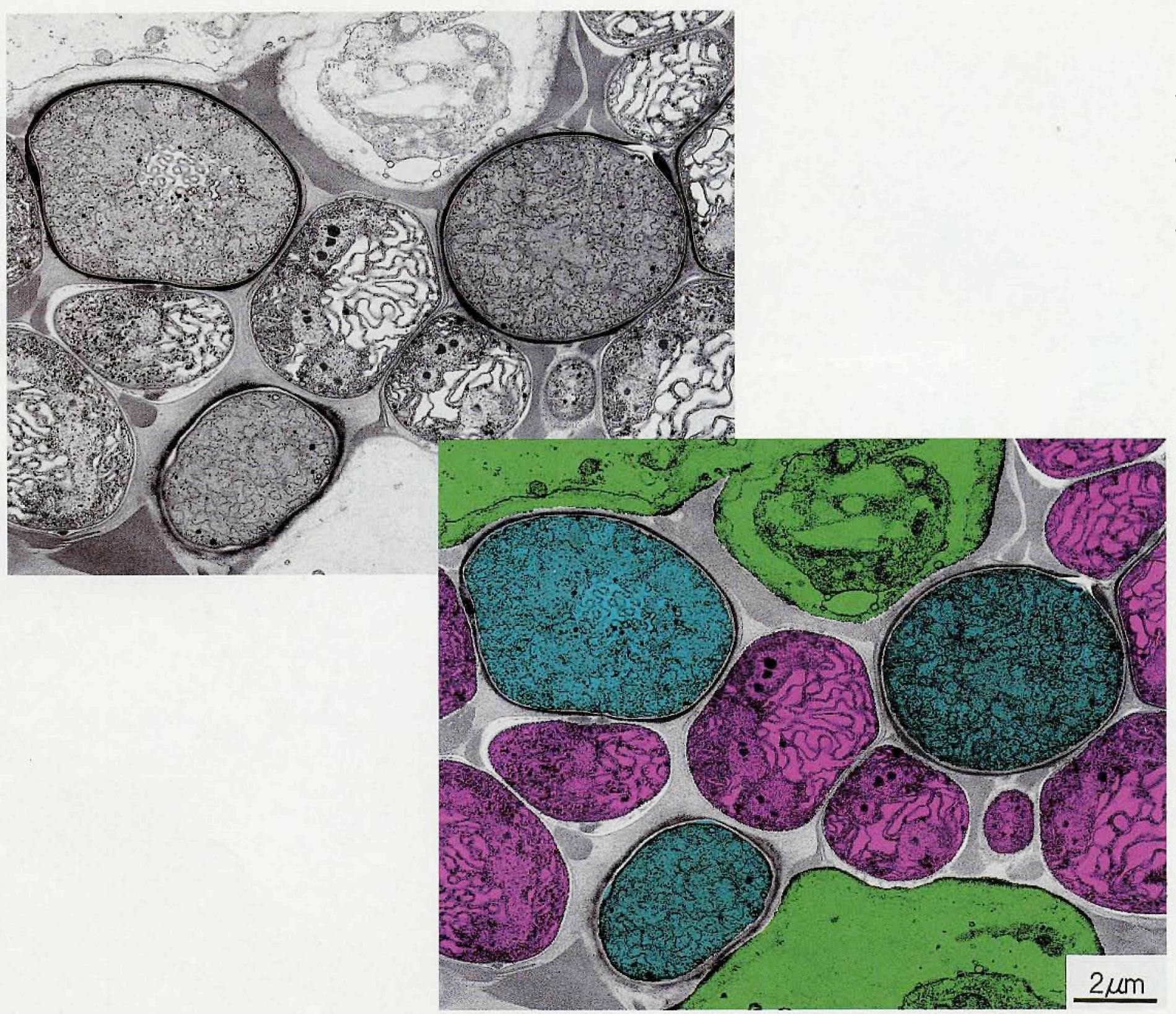




\section{The most}

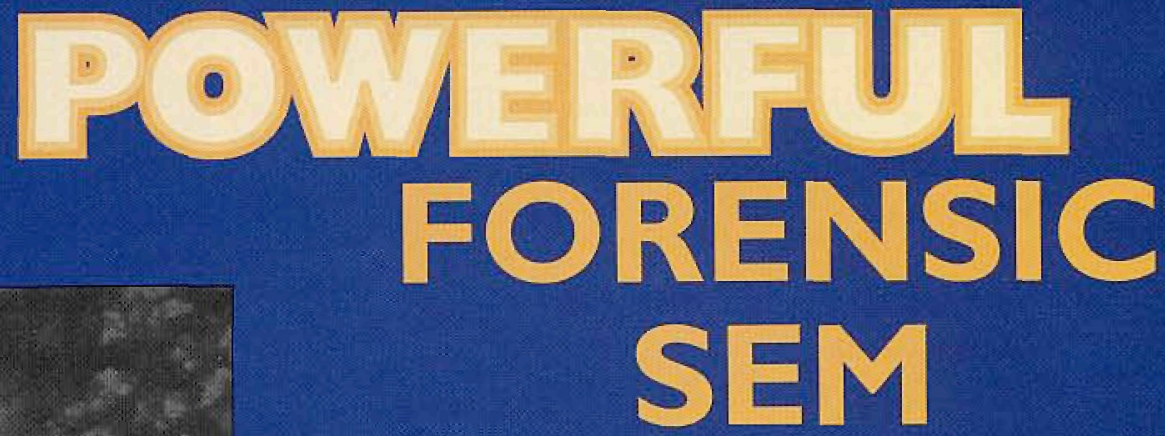

\section{on the Market}

5000x Philips XL30-DX4i-GSA particte

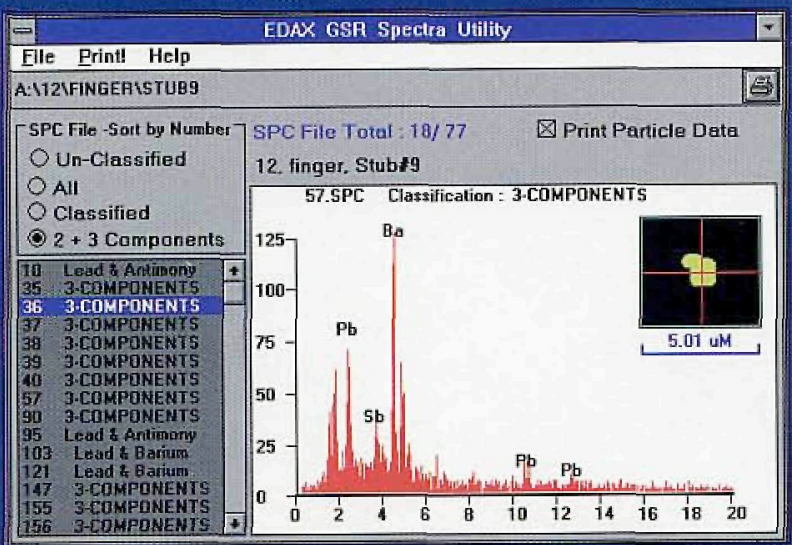

Our new $X L 30$ brings unique investigative capabilities to the forensic laboratory. This highly advanced scanning electron microscope can be equipped with a dedicated module for the automatic detection and quantification of gun shot residue (GSR). Other features: embedded EDX capability for element and chemical identification, digital data/image storage and excellent low magnification imaging.

The Controlled Pressure (CP) option allows imaging and analysis of uncoated specimens by applying backscatter detection in a gaseous environment.

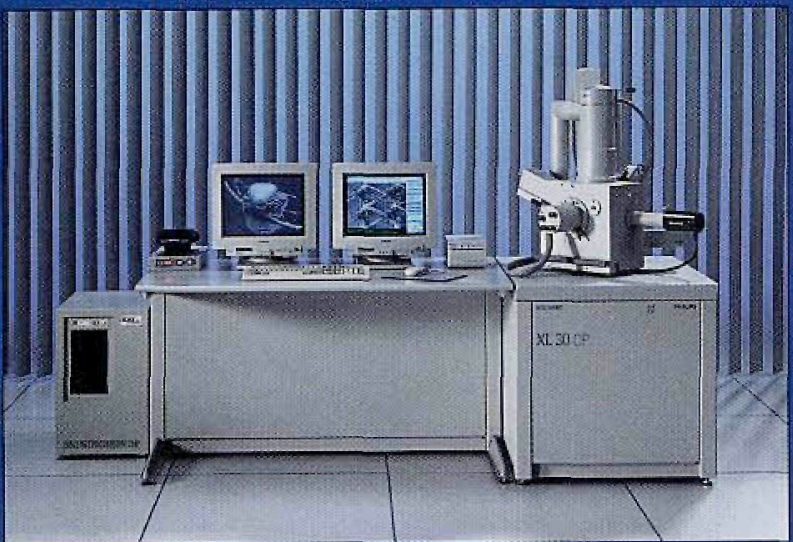

This unique combination exemplifies the perfect integration of different detection technologies in one single system.

So if you are looking for the best SEM for forensic science applications, here's a clue: investigate the Philips Electron Optics XL30. You'll find it's the right solution.

Philips Electronic Instruments Inc. 85, McKee Drive 


\section{ATOMIC FORCE MICROSCOPY FOR BIOLOGISTS}

Stephen W. Carmichael, ${ }_{1}^{1}$ Mayo Clinic

Atomic force microscopy (AFM) has proven to be very useful to material scientists and physicists. Biologists are only beginning to utilize the potential of this methodology. In a recent article, Tatsuo Ushiki, Jiro Hitomi, Shigeaki Ogura, Takeshi Umemoto, and Masatsugu Shigeno reviewed the applications of AFM to biologic studies.

They began by reviewing the basic principles of AFM, emphasizing the value of the non-contact mode for visualizing the relatively "soft" surface of biologic specimens. They presented some examples of biologic images: DNA, chromosomes, and collagen fibrils. The specimens examined with the AFM did not need to be coated, theoretically offering a view with a smaller potential for artifacts. AFM images have the advantage of containing quantitative information about the sample height. For example, analysis of an AFM image of a collagen fibril indicated the presence of a shallow depression in the middle of ridges on the fibril and sometimes a slight elevation within the grooves. It is possible that these features are a result of the air-drying used in the preparation of these fibrils. Ushiki et al. pointed out that when they are able to visualize collagen fibrils under physiologic conditions (i.e., in saline), the picture may be different.

Ushiki et al. also sectioned some cells and then extracted the embedding medium from the specimens. It was noted that the image is comparable to a low-power transmission electron micrograph.

Perhaps the most exciting images were those made of living cells. Due to limitations in the ability of the AFM to detect relatively large variations in height (limited $\mathrm{z}$ scan range), flat cells are better suited for examination. Images of a cultured cell (A549 cell from a human pulmonary adenocarcinoma) in the contact mode revealed structures appearing to represent the cytoskeleton just under the surface of the plasma membrane. It was suggested that the scanning probe deformed the surface sufficiently to outline underlying structures. When the cell was fixed with glutaraldehyde, the contour of the cytoskeletal elements disappeared and numerous granular elevations appeared corresponding to microvilli.

A time-lapse series of AFM images taken about 4 minutes apart demonstrated cell movement. Cultured human esophageal squamous cell carcinoma cells were clearly shown to have movement of their lamellipodia and a rearrangement of underlying cytoskeletal elements could be seen.

Ushiki et al. also pointed out that other scanning probe microscopes are proving to be useful in biologic studies. These methodologies include the friction force microscope, the magnetic force microscope, force modulating imaging, and the scanning near-field optical/atomic force microscope. Using this latter instrument, the authors have succeeded in observing cultured cells with fluorescing cytoskeletal elements.

It is clear that the AFM, along with its scanning probe cousins, will make important contributions to our understanding of biologic morphology. ${ }^{2}$

1. The author gratefully acknowledges Dr. Tatsuo Ushiki, for reviewing this article.

2. Ushiki, T., J. Hitomi, S. Ogura, T. Umemoto, and M. Shigeno, Atomic force microscopy in histology and cytology, Arch. Histol. Ctyol., 59:421-431, 1996.

3. Note added in proof. While this column was being prepared, an article by Stefan Schneider et al, appeared in PNAS, lending credence to this concluding statement. This article will be the subject of next month's column.

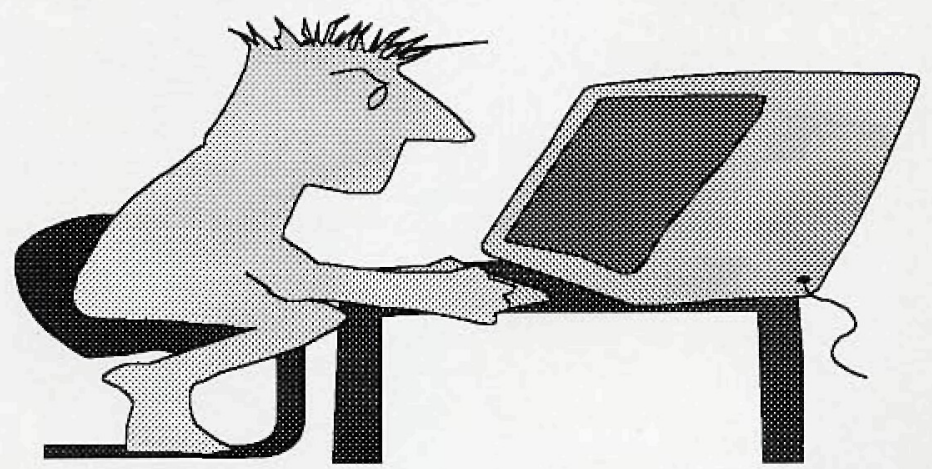

\section{Front Page Image}

\section{Moss}

The TEM micrograph on the front cover shows a sectional view of the thallus of Anthoceros subbrevis (one type of moss) and the symbiont, Cyanophyta (blue-green algae). The original greyscale image was taken using the new Hitachi $\mathrm{H}-7500$ PC-TEM and recorded onto a $\mathbf{2 5} \mu \mathrm{m}$-pixel digital imaging Plate. The image was then printed with a Fuji photo-grade Pictrography $\mathbf{3 0 0 0}$ printer. The accompanying colored image is of the same area, but the cells have been colored to highlight different internal structures which represent active conditions of each cell. The green areas represent the thalus of Anthoceros subbrevis while the blue and red areas indicate the location of the Cyanophyta cells. Data courtesy of Professor H. Matsushima, Saitama University, Japan

Specimen:

Acc. Voltage: Magnification:
Anthoceros subbrevis

$100 \mathrm{kV}$ $x 8,500$ (final), $x 5,000$ (direct)

\section{mIOROSCOPY TODAY}

A monthly newsletter dedicated to the unique interests in microscopy and mailed to some 8,000 microscopists worldwide - at no cost in the U.S. and with a modest international charge.

PO Box 620122, Middleton, WI 53562 - Tel.: (608)836-1970 - Fax: (608)836-1969 - eMail: MicroToday@aol.com

http://www.mictoscopy-today.com

Copyright in 1997 by Today Enterprises. All rights reserved.

Don Grimes, Editor 